Paper:

\title{
Study on the Adaptation with Learning About the Environment: The Case of Post-Acquisition Integration
}

\author{
Jing Su*, Mohsen Jafari Songhori**, and Takao Terano* \\ *Tokyo Institute of Technology \\ 4259 Nagatsuta-cho, Midori-ku, Yokohama, Kanagawa 226-8502, Japan \\ E-mail: sujing-ch@hotmail.com, tterano@computer.org \\ ** Department of Health Technology and Services Research, Technical Medical Center, University of Twente \\ Ravelijn 5151, P.O. Box 217, 7500 AE Enschede, The Netherlands \\ E-mail: mj2417@gmail.com \\ [Received December 22, 2017; accepted June 8, 2018]
}

\begin{abstract}
Organizations can be considered as complex systems that can adapt to their changing environment. In this work, we study a complex system adapting to an unfamiliar environment with learning; this is grounded in the context of the post-acquisition integration of the companies. More specifically, we conceptualize post-acquisition integration from the perspective of behavioral theory as a reason for the environmental changes to the firms (agents). We studied the adaptation of these complex systems and we propose a coupled learning method over the NK landscape. The simulation results show that the initial perceptions of the agents regarding the new task environment can be quite influential to the performance of the entire system during the adaptation process. Correct initial perceptions can help the system to quickly achieve high performance, whereas incorrect initial perceptions may prevent the system from reaching high performance. Lack of initial perceptions could lead to a slow yet robust adaptation process with a moderate level of performance. Moreover, certain other factors, such as the sensitivity to the feedback from the environment, the incentive of the system for exploration, and the learning frequency, may have different impact on the adaptation and performance of the system.
\end{abstract}

Keywords: complex systems, adaptation, learning, agent-based simulation, post-acquisition integration

\section{Introduction}

Complex adaptive systems (CAS) are systems that have a large number of components that interact and adapt themselves to the problems that arise from their surroundings $[1,2]$. Research endeavors using the CAS perspective have been undertaken in many fields, such as physics, biology, economics, engineering, and organization theory [3].

Typically, organizations can be considered as complex systems [4]. For instance, according to the behavioral the- ory of the firm [5], the business goal of a company can be conceptualized as a search for good strategies to obtain high economic payoffs, where a strategy can be conceptualized as a series of choices, e.g., whether to expand its market, develop a new product, make a personnel change, etc. These choices may be highly interdependent from one another; thus the change of one choice is likely to result in the changes of others. The complex interdependencies among these choices make the company a complex system.

In reality, the external environment of a company can often change for many reasons, which means that the economic payoffs resulting from the choices of the company are subject to change. Thus, the company has to adapt to the new environment to continuously obtain high payoffs. The NK model proposed by Kauffman [6] is widely used to model the external environment of the organizations [7-9] because it allows researchers to maintain control over the interactions among the components of the system and facilitates the modeling of the environmental complexity and environmental change [7]. Several academic studies discuss the adaptation of companies and use the NK model to build the changing environment (landscape); however, most of them model the adaptation process only through a search process without considering the lack of knowledge of the company about the changing environment. Considering the bounded rationality [10], a company may have limited knowledge about the new environment; this could be highly influential to its search performance and, consequently, its adaptation performance. Thus, companies may need to learn about the new environment in order to adapt to it.

One of the reasons for environmental change can be mergers and acquisitions (M\&A). As two companies merge together, their business, strategies or decisions may become interrelated and affect one another; consequently, their environment will change. Thus, the two merged companies may need to adapt to the new environment, particularly during the post-acquisition integration phase. Nevertheless, most of the existing research regarding the post-acquisition integration consists of empirical works that have mainly been focused on the procedures of the in- 
tegration. There are only scarce research works that have been focused on the adaptation process of the companies during the integration phase.

Therefore, in this work, we will study the learning and adaptation of a complex system in an unfamiliar environment using the case of the post-acquisition integration of companies. More specifically, this work makes two main contributions. Regarding the methodology, we propose a coupled learning method for adaptation over the NK landscape while considering the bounded rationality in recognizing the environmental change. In the context of M\&A, we will model and discuss the problem of postacquisition integration of two companies from the perspective of CAS, and we will build an agent-based model that applies the proposed learning method to study the factors that influence the performance of the companies during the post-acquisition integration phase. A review of related works will be presented in the following section. The model will be described in Section 3, followed by the simulation results in Section 4. Finally, the conclusions will be summarized in Section 5.

\section{Literature Review}

In the field of organizational theory and management science, several researchers have studied the adaptation behaviors of organizations. For instance, Levinthal [11] modeled the adaptation process of an organization through the evolution of a population of the organizational forms, and discussed the impact of environmental complexity on the adaptation of the organization. Yi et al. [9] examined how inertia in routines influences the process of organizational adaptation. Uotila [7] examined how the environmental turbulence and complexity influence the temporal patterns of incremental and radical organizational change. Certain studies tend to discuss the relationships between the adaptation of the organization and the environmental properties, such as complexity [12], or the frequency and magnitude of the environmental dynamism [13]. On the other hand, other studies have been focused on the behaviors of the adaptation of the organization in the dynamic environment $[9,14,15]$. However, most of these studies have been focused only on the adaptation of one single company. Only rarely has research been conducted on the adaptation during the M\&A process.

More specifically, the NK model is a popular platform for the modeling of complex adaptive systems in the field of organizational theory as well. It is widely used for the modeling of the organizational structures $[16,17]$ or of the external environment of the organizations [7-9, 12, 14, 15] because it allows modelers to control the complexity and the dynamic of the system. According to the mechanism of the NK model, the environmental dynamic can be defined as the change in the mapping from the actions of the organization to the performance payoffs; this mapping is determined by the interactions between the aforementioned actions and the performance contributions of each action. Nevertheless, in most studies, the environmental dynamic has been modeled as the change in the performance contributions of the actions $[7-9,15]$; there are only few studies in which the environmental turbulence has been modeled as the change in both the interactions and performance contributions [14].

Furthermore, the bounded rationality [10] - which indicates the limited rationality of individuals (or agents) in decision making owing to the limited knowledge and information, cognitive limitations, and available time to make the decision - is often considered by the modelers from various perspectives in the studies of behavioral theory. Aggarwal et al. [18] and Claussen et al. [8] considered the bounded rationality of the limited authority of each company (department) in conducting search within the collaboration (to search only on its own subcomponent). Csaszar et al. [19] and Knudsen et al. [20] modeled the bounded rationality as the ability of the decision makers to evaluate or screen the alternatives owing to the effect of different domains of expertise. Mihm et al. [21] implemented their search model, in which they introduced the bounded rationality of the search authorities of the decision makers, as well as the frequency of communications through which the decision makers could obtain the necessary information to make proper choices. Nonetheless, only few researchers consider the bounded rationality in recognizing how the environment has changed, despite the fact that it is a crucial factor that could affect the search process of the agents and, consequently, the adaptation of the organization.

In the literature, there is a great interest in postacquisition integration because it was found to have a great effect on the success of M\&A [22]. Birkinshaw et al. [23] distinguished the post-acquisition integration in task integration and human integration. Human integration concerns generating satisfaction and shared identity among the employees, whereas task integration focuses on value creation and operational synergies. Regarding the task integration, in certain studies it has been argued that the value creation or the performance of the company are affected by the level of integration [24,25], whereas certain other studies have been focused on the influence of integration speed [26]. In addition to task integration and human integration, knowledge learning, sharing, and transfer during the integration process have been found to be influential factors to the success of acquisitions as well [27-30]. However, most works that have been focused on the study of post-acquisition integration are empirical works, and the emphasis has been placed on the procedures of the integration. There are very few research works in which the adaptation of companies to the new environment has been discussed.

Based on the aforementioned literature review, we conceptualized the post-acquisition integration as the reason of an environmental change from the perspective of behavioral theory and complex adaptive systems. Furthermore, we studied the adaptation of the complex system by proposing a coupled learning method over the interaction matrix of the NK landscape while considering the 
bounded rationality in the recognition of environmental change.

\section{Model}

In this section, we will describe our model for the postacquisition integration of two firms. More specifically, we will model two companies as two agents and the operations thereof as searching for good strategies in their original environment. After the acquisition, the two companies will merge together, and they will become two subsystems of the entire organization. Accordingly, their strategies become correlated to one another; thus, their environment would change. Considering the bounded rationality, both companies may potentially be unfamiliar with the new environment owing to their limited knowledge they have about one another (e.g., business, techniques, etc.). However, the two companies could adapt to the new environment by adopting a collaborative search behavior and a coupled learning behavior. It should be noted that we will discuss the behavior of the system with a focus on the adaptation process after the environmental change rather than during the environmental change. The details of the model will be described in terms of four aspects: the environmental changes during the post-acquisition integration phase, the limited knowledge of the companies about the new environment, the collaborative search behavior of the companies, and the coupled learning behavior of the companies.

\subsection{Environmental Changes During the Post- Acquisition Integration Phase}

\subsubsection{Original Task Environments of Two Companies}

The NK model conceives the target problem in terms of a high-dimensional fitness landscape. Each component of the system constitutes a horizontal dimension, and the fitness outcome of the system constitutes the vertical dimension, thus creating a landscape function [31]. More specifically, the mapping from the horizontal dimensions to the fitness outcome is controlled by the interactions and the fitness contributions of the horizontal dimensions, rather than by a particular mathematical function. Parameter $N$ of the "NK" controls the number of the system components (i.e., horizontal dimensions); parameter $K$ of the "NK" controls the number of interactions that each component has with other components.

More specifically, the target problem can be defined as an $N$-digit string of $\boldsymbol{s}=\left\{s_{1} s_{2} \ldots s_{N}\right\}$, where each of the $s_{i}$ denotes a component of the target problem. Each component makes a contribution $C_{i}$ to the fitness of the entire string, and $C_{i}$ depends on the value of $s_{i}$, as well as the values of $K$ other components that have interactions with $s_{i}$, which are denoted as $\left\{s_{j}^{i}\right\}=$ $\left\{s_{1}^{i}, s_{2}^{i}, \ldots, s_{j}^{i}, \ldots, s_{K}^{i}\right\}$. Hence, this contribution can be denoted as $C_{i}=C_{i}\left(s_{i} ;\left\{s_{j}^{i}\right\}\right)$. Then, the overall fitness of the string can be evaluated as the average of the contributions of the components, and can be expressed as Eq. (1).

$$
F(s)=\frac{1}{N} \sum_{i=1}^{N} C_{i}\left(s_{i} ;\left\{s_{j}^{i}\right\}\right) \text {. . . . . . . . . . }
$$

Consequently, the complexity of the system, the ruggedness of the landscape, and the shape of the landscape can be controlled by the interactions and the fitness contributions of the components.

The task environment of the company can be modeled as an NK landscape. More specifically, the operational strategy of the company can be modeled as a series of binary decisions about how to configure different activities. For instance, the company has to decide whether to expand its market, whether to develop a new product, etc. Thus, we define an $N$-digit string of $\boldsymbol{d}=\left\{d_{1} d_{2} \ldots d_{N}\right\}$ to represent the strategy of a company. Element $d_{i}$ can receive the value of either 0 or 1 , thus indicating the decision of an activity (e.g., whether to approve a project or not). Each configuration of a strategy has a corresponding fitness value. The fitness value can be considered as the economic payoff of that strategy in the reality; moreover, in our model, it can be used for the measurement of the performance of the company.

According to the previously described mechanism of the NK model, each decision $d_{i}$ has a contribution to the fitness of the strategy, denoted as $C_{i}=C_{i}\left(d_{i} ;\left\{d_{j}^{i}\right\}\right)$, which is affected by the choice (value) of the focal decision, as well as by the choices of certain other relevant decisions. The exact set of relevant decisions $\left\{d_{j}^{i}\right\}$ for each $C_{i}$ is determined by the interactions between the decisions; these interactions can be integrated to an interaction matrix. In this research, to simplify the model without loss of generality, we adopted a random pattern for each of the original task environment of the companies. Fig. 1 shows an example of the interaction matrices of the environments of the two companies, where mark "Y" indicates the focal decision and mark " $\mathrm{x}$ " indicates the interaction between the exact decision and the focal one. Changing the value of either $d_{i}$ or of any relevant decision $d_{j}^{i}$ may result in a different contribution value $C_{i}$. These contribution values are independently drawn at random from a uniform $U[0,1]$ distribution.

Finally, the overall fitness associated with an exact configuration of the decisions can be evaluated via Eq. (2). A higher fitness value indicates better strategy. With all possible strategy configurations and corresponding fitness values, the original landscapes of the environments of the two companies can be determined.

$$
F(\boldsymbol{d})=\frac{1}{N} \sum_{i=1}^{N} C_{i}\left(d_{i} ;\left\{d_{j}^{i}\right\}\right)
$$

\subsubsection{Environmental Change After the Acquisition}

Environmental changes can be commonly defined as changes in how strategic actions impact the performance outcomes [13]. Thus, it can be defined as the change in the mapping between the strategies and their fitness values 

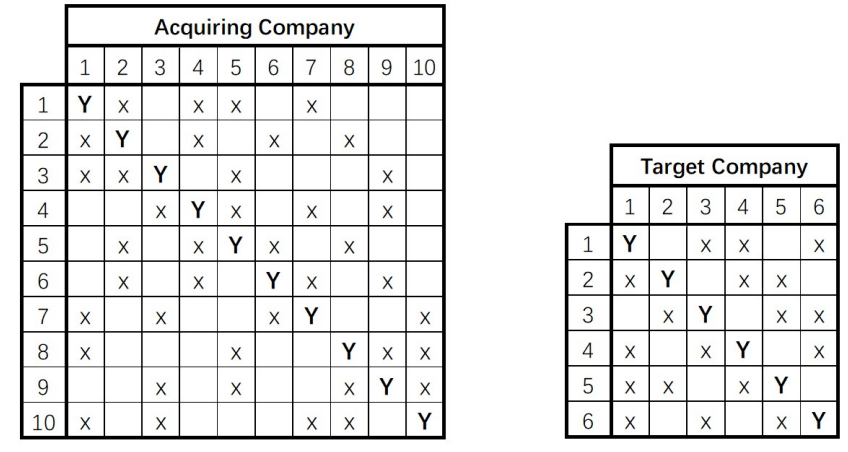

Fig. 1. Interaction matrices of the original task environment of two companies (Acquiring company: $N=10, K=4$, Target company: $N=6, K=3$ ).

(i.e., the change in landscapes). In this research, we considered the acquisition case that two companies combine their respective businesses and become interdependent. ${ }^{1}$ In the following sections, we will use the term "organization" to refer to the entire company after the acquisition; the terms "the acquiring company" (or "the acquirer") and "the target company" (or "the target") will be used to refer to the subsystems of the former acquiring company and of the former target company, respectively.

More specifically, we define the strategy of the organization after the acquisition as the combination of the original strategies of each of the two companies. As the businesses become merged, we assume that some of the decisions of the strategies of the two companies become correlated in the form of emerging interactions between them. Consequently, the contribution of each decision, hence the fitness landscape, could change as well. In our research, we employed the work of Claussen [8] to model the interaction matrix of the new environment. Fig. 2 shows an example of the new interaction matrix that refers to the examples illustrated in Fig. 1. The integrated strategy of the organization contains $\left(N_{A}+N_{T}\right)$ decisions; the former acquirer accounts for $N_{A}$ decisions and the former target accounts for $N_{T}$ decisions. For each decision, we assumed that the original interactions within the company from which it originated would remain unchanged during the acquisition; $K_{B}$ is the number of new interactions with the decisions of the other company that are expected to emerge. Therefore, the intra-firm interaction patterns at the upper left area and lower right area are consistent with the patterns in the left and right graphs of Fig. 1, respectively, whereas the inter-firm interactions appear at the upper right area and lower left area.

As the interaction matrix would change, the contribution of each decision to the fitness of strategy would change as well. The new environment landscape will be derived from the two original ones and can be ob-

1. This type of acquisition may occur when a core company wishes to explore certain new functions on its products or to combine its own business with other businesses, yet it has little knowledge of the exact fields. Then, the core company may cover these shortages by acquiring a peripheral company that has expertise in these fields.

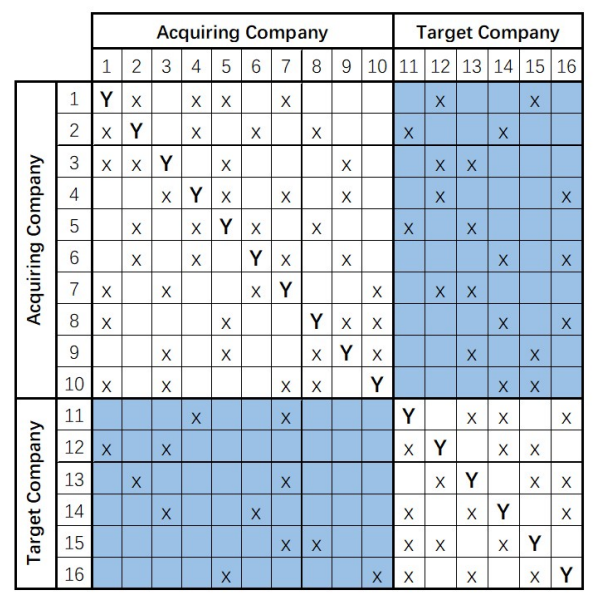

Fig. 2. Interaction matrix of the new environment after the acquisition $\left(N_{A}=10, K_{A}=4, N_{T}=6, K_{T}=3, K_{B}=2\right)$.

tained from the correlations between the original and the new contributions of each decision. More specifically, for each decision $d_{i}$, the new contribution is denoted as $C_{i}^{\prime}=C_{i}^{\prime}\left(d_{i} ;\left\{d_{j}^{i}\right\},\left\{d_{l}^{i}\right\}\right)$ and the original contribution is denoted as $C_{i}=C_{i}\left(d_{i} ;\left\{d_{j}^{i}\right\}\right)$, where $\left\{d_{j}^{i}\right\}$ indicates the original interactions and $\left\{d_{l}^{i}\right\}$ indicates the new interactions. Then, $C_{i}^{\prime}$ is drawn at random from a triangular distribution $\operatorname{Tr}\left(0, C_{i}, 1\right)$, where 0 is lower limit, 1 is upper limit, and $C_{i}$ is the mode. For instance, the original contribution of decision No.6 is $C_{6}=C_{6}\left(d_{6} ;\left\{d_{2} d_{4} d_{7} d_{9}\right\}\right)$ according to the left graph of Fig. 1; then, it becomes $C_{6}^{\prime}=$ $C_{6}^{\prime}\left(d_{6} ;\left\{d_{2} d_{4} d_{7} d_{9} ; d_{14} d_{16}\right\}\right)$ according to Fig. 2. Therefore, each configuration of $\left(d_{6} ;\left\{d_{2} d_{4} d_{7} d_{9}\right\}\right)$ will produce four new configurations owing to the new interactions of $d_{6}$ with $d_{14}$ and $d_{16}$. The new contribution of each new configuration will be generated following the distribution of $\operatorname{Tr}\left(0, C_{6}, 1\right)$.

With the new interaction matrix and the new contributions, we can evaluate the fitness of the strategies of the entire organization and generate a new environment landscape for the organization. This landscape is identified as a new task environment in this research.

\subsection{The Limited Knowledge of the Companies About the New Task Environment}

Considering the bounded rationality, each company may have limited knowledge about how the decisions, activities, and/or business of the other company could affect its own even after the knowledge transfer, because certain "tacit knowledge" is difficult to be transferred during the acquisition [27, 29]. In our model, this limited knowledge can be represented by the partially correct perception of the interactions between the decisions. We assumed that each company has a perceived interaction matrix with partially correct interactions regarding the "new task environment". This perceived interaction matrix could affect 
the contribution evaluation of the decisions and, consequently, the fitness evaluation of the strategy. Therefore, the decision making of the organization could potentially be affected by the perception that each company has of the new environment. In the following sections, we will introduce the perceived environment landscape of each company in terms of the interaction matrix and the contributions of the decisions.

\subsubsection{Perceived Interaction Matrix}

We assumed that the perceived interaction matrix of each company is determined by the knowledge of its own subsystem and the knowledge of the other subsystem, which has been shared by the other company. As the two subsystems are symmetric in the organization, we will introduce the details on the perceived interaction matrix from the perspective of the acquiring company.

Figure 3 shows an example of the perceived interaction matrix of the acquiring company. The upper left and upper right areas show the knowledge that the acquiring company has of its own subsystem, which contains decisions No.1 through 10. We assume that the acquiring company has full knowledge of the intra-firm interactions; hence, the pattern at the upper left area is consistent with the one of the real environment, as shown in Fig. 2. For the inter-firm interactions (i.e., the upper right area), we assume that the company has $X$ (percentage) of the correct knowledge, which is indicated by the cells in dark color. Then, the remaining $1-X$ of interactions will be determined from the decisions indicated by the cells in light color. The aforementioned $X$ of interactions will be randomly determined at the beginning of the simulation.

We assume that the company knows that the number of inter-firm interactions is $K_{B}$ for each decision $d_{i}$. In addition, we assume that the company has correct knowledge of $K_{k}^{i}$ interactions. Then, for decision $d_{i}$, the company has to determine the remaining number of interactions, namely $K_{B}-K_{k}^{i}$, from $N_{T}-K_{k}^{i}$ candidates. For instance, for decision No.1, the company has to determine one interaction from candidates No.11, 12, 13, 14, and 16 (i.e., $K_{B}=2, K_{k}^{1}=1$ ), whereas, for decision No.2, it has to determine two interactions from the candidates No.11 to 16 (i.e., $K_{B}=2, K_{k}^{2}=0$ ). However, the company does not have to determine any interaction for decision No.5 because it has full knowledge about its interactions (i.e., $K_{B}=2, K_{k}^{5}=2$ ).

Although the company is not aware of certain interactions regarding the focal decision, it may have a certain perception about which candidate(s) is (are) likely to be the interaction(s). We modeled this perception as a set of expected payoffs and we will refer to it as "belief". For instance, regarding decision No.1 in Fig. 3, the company has a belief about the expected payoffs for interaction candidates No.11, 12, 13, 14, and 16, which are denoted as $\left\{w_{11}^{1}, w_{12}^{1}, w_{13}^{1}, w_{14}^{1}, w_{16}^{1}\right\}$. Then, the company can determine the interaction for decision No.1 based on these expected payoffs.

2. The "partially correct knowledge" of the inter-firm interactions has been

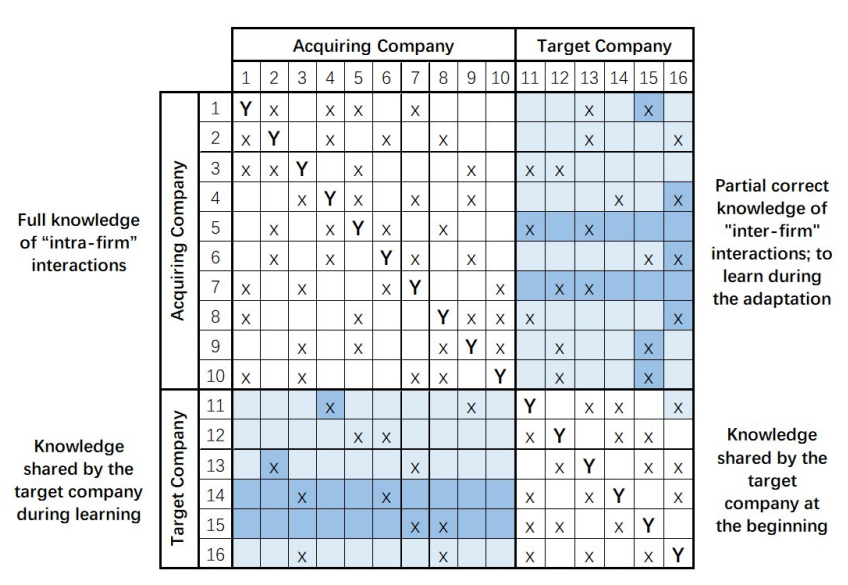

Fig. 3. The perceived interaction matrix of the acquiring company. ${ }^{2}$

In general, for decision $d_{i}$, the company has a belief of the expected payoffs $\left\{w_{m_{1}}^{i}, w_{m_{2}}^{i}, \ldots, w_{m_{s}}^{i}\right\}$ pertaining to the corresponding $s$ interaction candidates, where $m_{1} \sim m_{s}$ denote the identity of the candidates. Then, the probability of selecting any of the candidates is linked to these expected payoffs by means of the softmax function which is widely used in the reinforcement learning process $[32,33]$. More specifically, the probability of selecting candidate $m_{j}$ is

$$
p_{m_{j}}^{i}=\frac{e^{\frac{w_{m_{j}}^{i}}{\tau}}}{\sum_{r=1}^{s} e^{\frac{w_{m_{r}}^{i}}{\tau}}}
$$

In Eq. (3), $w_{m_{j}}^{i}$ indicates the expected payoff of candidate $m_{j}$ for decision $d_{i}$. Parameter $\tau$ controls the exploration level for searching for the potential interactions [32,34]. High $\tau$ values result in equal likelihood for selecting any of the candidates, whereas low $\tau$ values result in a higher probability of selecting the candidates with higher expected payoffs. Finally, to determine the selection of any candidate, the roulette wheel mechanism was used in conjunction with the probabilities derived from Eq. (3).

The lower left and lower right areas in Fig. 3 show the knowledge shared by the target company, which contains the interactions of decisions No.11 through 16. Considering that two companies would share knowledge of their business, technologies, and policies to one another during the acquisition, we assume that the target company will share the knowledge of its intra-firm interactions to the acquirer at the beginning of the simulation (the lower right area in Fig. 3). Considering that the two companies may communicate through meetings, we assume that the target company will share the knowledge of the inter-

indicated at the lower left area and the upper right area of the graph in the same manner. However, the knowledge and the authority of learning over the lower left area belong to the target company. For the acquiring company, all inter-firm interactions in this area will be shared by the target company during the learning process. 
firm interactions of its own subsystem to the acquirer after each time of determination (the lower left area).

Consequently, the acquiring company can obtain a perceived interaction matrix with its original knowledge of the intra-firm interactions, the inter-firm interaction that has been determined according to the belief of expected payoffs, and the knowledge shared by the target company. Similar to the acquirer, the target company has a perceived interaction matrix of the environment landscape containing its own knowledge and the knowledge shared by the acquirer.

\subsubsection{Evaluation of Contributions Based on the Per- ceived Interaction Matrix}

With the perceived interaction matrix, the companies can first evaluate the contributions of each decision and then, the fitness of the strategy string. We assume that each company evaluates the contributions and the fitness independently. For each decision, the company is able to properly evaluate its contribution (i.e., its contribution is equal to the real contribution of the new environment), if all of its interactions have been correctly perceived (i.e., consistent with the real interaction matrix of the new environment). If the perceived interactions are not consistent with the actual ones, the company cannot properly evaluate the contributions.

Figure 4 shows the mechanism of the contribution evaluation in our model. For example, decision No. $i$ has real interactions with decisions No.3 and No.10; however, the company perceives interactions with decisions No.3 and No.7. Then, the evaluation of the company regarding the contribution will be affected by the incorrect perception of the interactions. More specifically, the possible configurations of decision $\left(d_{i} ;\left\{d_{3} d_{10}\right\}\right)$ and the corresponding contributions are shown in the leftmost box of Fig. 4 as the real contributions with the real interactions of decision $d_{i}$. As each decision can receive two values $(0$ or 1$)$, there are 8 possible configurations of $\left(d_{i} ;\left\{d_{3} d_{10}\right\}\right)$, which are shown in the first column of the leftmost box. Because the company has mistakenly perceived one of the interactions as $d_{7}$ rather than $d_{10}$, the evaluation of the contributions of $d_{i}$ will be affected by this incorrect perception. We define the evaluated contribution (shown in the rightmost box of Fig. 4) as the contribution with correctly perceived interactions (shown in the middle box of Fig. 4) plus a noise denoted as $\widetilde{n}$. For instance, when the perceived $\left(d_{i} ;\left\{d_{3} d_{7}\right\}\right)$ receives the values (the configuration) of (000) or (001), the evaluated contribution will be $C_{-} 1+\widetilde{n}$, where $C_{-} 1$ is the average contribution when $\left(d_{i} ;\left\{d_{3}\right\}\right)$ receives the values of $(00)$. This average contribution, $C_{-} 1=(C 1+C 2) / 2$, can be derived from the real contributions, namely $C 1$ and $C 2$, when $\left(d_{i} ;\left\{d_{3} d_{10}\right\}\right)$ receives the values of (000) and (001).

Noise $\widetilde{n}$ is generated independently for each configuration of decisions and follows a normal distribution with a standard deviation that is correlated to the incorrect rate of perceived interactions. In the example of Fig. 4, the incorrect rate is 0.5 (one error among the two interactions); thus

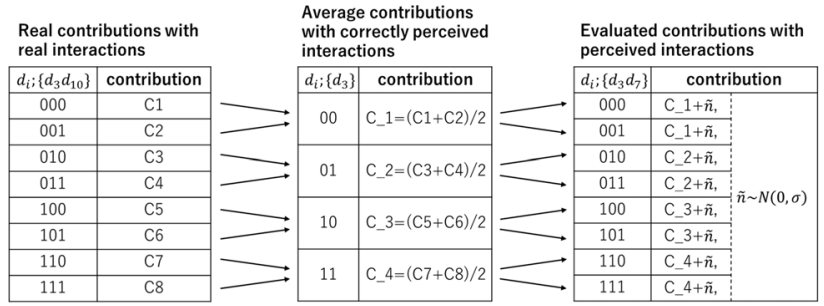

Fig. 4. Mechanism of generating contributions with perceived interactions.

the standard deviation is defined as $\sigma=0.1 * 0.5=0.05$. Parameter 0.1 is set to adjust the magnitude. Therefore, more accurate contributions are obtained with less incorrect perceived interactions. Moreover, the contributions of each decision are time invariant, with the same interaction pattern.

With the perceived interaction matrix and the corresponding evaluated contributions, each company can evaluate the fitness of strategies to obtain a perceived environment landscape.

\subsection{Collaborative Search Behavior of the Compa- nies}

In addition to the external environment construction and the definition of the limited knowledge of the company, the search behavior and learning behavior of the companies will be clarified respectively in this section and the next section to describe the manner in which the organization adapts to the new environment.

We define both companies as decision makers. The acquiring company has authority over decisions No.1 through 10, whereas the target company has authority over decisions No.11 through 16. The search behavior of the organization is carried out by the collaboration of the two companies.

During each time period, the two companies conduct local search by making a choice regarding whether to alter the value of the decision elements of the strategy string (i.e., to change a decision from 0 to 1 , or vice versa). More specifically, each company can randomly select one of the decisions in its subsystem and alter its value based on the current strategy to obtain an alternative. Then, the company will evaluate the fitness of the alternative and approve it as a proposal if the fitness is higher than the one of the current strategy. On the contrary, the company will propose the current strategy if the fitness of the alternative is lower. It should be noted that each company evaluates the fitness based on its own perceived environment landscapes, which are derived via the mechanism described in the previous section.

Particularly, we consider three collaboration types with different decision-making order [18]: (I) the acquiring company searches first, (II) the target company searches first, and (III) the two companies search simultaneously. Collaboration Type I indicates the case in which the acquiring company has a high priority to make decisions. 
During each time period, the acquiring company conducts its local search based on the current strategy. Then, the target company conducts its local search based on the proposal of the acquirer. Finally, the new strategy proposed by the target company will be implemented. Collaboration Type II indicates the case in which the acquiring company has the authority to make the final decision before the approval of the strategy. The search sequence is opposite to that of collaboration Type I. Collaboration Type III indicates the case in which the two companies are relatively independent in the decision-making process. During each time period, both companies conduct local search simultaneously based on the current strategy and introduce their proposals. Then, the strategy that will be implemented would be the combination of the corresponding sub-components of the proposals of the two companies (sub-component with decisions No.1 through 10 from the proposal of the acquirer and sub-component with decisions No.11 through 16 from the proposal of the target).

\subsection{Coupled Learning Behavior of the Companies}

Because both companies have incomplete knowledge about the inter-firm interactions, they may adapt to the new environment through a "try and learn" behavior. We assume that the organization conducts one trial every $T_{L}$ time periods and randomly selects $n_{L}$ decisions to execute a reinforcement learning process. Parameter $n_{L}$ represents the incentive of the exploration; low $n_{L}$ value indicates a cautious organization, whereas a high $n_{L}$ value indicates an adventurous organization.

In each trial, we assume there are $n_{L}^{A}$ decisions that belong to the subsystem of the acquirer and $n_{L}^{T}$ decisions that belong to the subsystem of the target; hence, $n_{L}^{A}, n_{L}^{T} \in\left[0, n_{L}\right], n_{L}^{A}+n_{L}^{T}=n_{L}$. The acquiring (target) company determines the perceived interactions based on its "belief" of expected payoffs for each of the $n_{L}^{A}\left(n_{L}^{T}\right)$ decisions and maintains the interactions of other decisions unchanged. Then, the two companies exchange information pertaining to the determined interactions and update their perceptions of interaction matrix. In the following $T_{L}-1$ time periods, the companies conduct search by evaluating the fitness of strategies with their new interaction matrices. Ultimately, the companies update the expected payoffs of the determined interactions for each of the selected decisions according to the following rule:

$$
w_{j, t}^{i}=w_{j, t-T_{L}}^{i}+\phi\left(\overline{F(\boldsymbol{d})}-w_{j, t-T_{L}}^{i}\right) \ldots .
$$

In Eq. (4), $w_{j, t}^{i}$ indicates the updated expected payoff of interaction No. $j$ for decision No. $i$; $w_{j, t-T_{L}}^{i}$ indicates the previous expected payoff. $\overline{F(\boldsymbol{d})}$, which can be obtained via Eq. (5), indicates the average performance feedback (i.e., the real performance obtained from the real task environment) of the organization with strategy string $\boldsymbol{d}$ that results from the search behavior in each of the $T_{L}-1$ time periods.

$$
\overline{F(\boldsymbol{d})}=\frac{1}{T_{L}-1} \sum_{m=1}^{T_{L}-1} F(\boldsymbol{d})_{t-T_{L}+m} \ldots \ldots .
$$

Parameter $\phi \in[0,1]$, as a key parameter in reinforcement learning, represents the rate at which the expected payoffs are rewarded (or penalized) with the performance feedback. A high $\phi$ may indicate that the company is sensitive to recognizing and adapting to the feedback. This updating rule captures the two central features of reinforcement learning models: (i) the reward (penalty) of the expected payoff via $\phi$ captures the tendency to repeat actions that perform well, while preventing the repetition of actions that do not perform well; (ii) the previous expected payoff $w_{j, t-T_{L}}^{i}$ implicitly represents an aspiration level of performance that depends on the history of the past performance $[32,35]$.

Although the two companies conduct reinforcement learning within their own subsystem (i.e., the upper right and lower left areas in Fig. 3), their learning behavior will probably affect one another via the collaborative search result because the decision elements of the two subsystems are highly correlated to each other. Therefore, this learning behavior is referred to as coupled learning behavior.

\section{Simulation and Results}

In this section, we will introduce the experiments and show the results based on the model that we proposed in the previous section. More specifically, we will discuss the behavior of the organization in adapting to the unfamiliar environment from two aspects: learning behaviors and search behaviors. We will first introduce the basic settings of the NK landscapes and the simulation; then, the experimental setups and results will be described. We assume the strategy of the acquiring company contains $N_{A}=10$ decisions, each of which has interactions with $K_{A}=8$ other decisions. The strategy of the target company is composed of $N_{T}=6$ decisions, each of which has interactions with $K_{T}=4$ other decisions. Moreover, we assume that there are $K_{B}=2$ inter-firm interactions that emerge for each decision element of the organization during the acquisition process. Therefore, we can obtain the post-acquisition environment landscape of the organization according to the mechanism that has been previously described in the model section. All simulation results are the average of 200 runs over 50 different landscapes (4 runs over each landscape). For each run, we started the organization by placing it at a random point on the environment landscape, and we set the simulation time to be $T=1600$ time periods. Different scenarios with different parameter settings were designed for the experiments. However, here we will only present the representative results of several scenarios with certain parameter values. Other results that were found to be similar will not be presented in this paper; however, they are available upon the request. In addition, we conducted the experiments with several $K_{A}, K_{T}$ and $K_{B}$ settings that represent different complexities of the system. It was found that our results and conclusions were not influenced by the different configurations of the aforementioned three parameters. 


\subsection{The Impact of the Learning Behaviors on the Performance of Adaptation}

The first round of experiments was focused on how the learning behaviors of the organization would affect the organizational performance in the adaptation process. The relevant parameters can be divided into two categories according to their implications: (i) the initial knowledge of the agents about the interactions among the decisions, and (ii) the learning behaviors of the agents. The initial knowledge encompasses two parameters: the proportion of the prior knowledge about the inter-firm interactions, denoted as $X$, and the initial belief about the expected payoffs of the interaction candidates, denoted as $\left\{w_{m_{j}}^{i}\right\}, \forall i \in\left[1, N_{A}+N_{T}\right]$.

We set two levels of $X$, namely $X=80 \%$ and $X=20 \%$, to represent the case in which agents have good or poor knowledge about the correlations between two companies (or, in other words, the inter-firm interactions). As the beliefs about the expect payoffs of the interaction candidates are quite crucial to the determination of the perceived interaction matrix for each agent, we designed three types of initial beliefs to indicate three different initial conditions. To describe these types, decision No.2 will be presented as an example. First, we assumed that the summation of the expected payoffs for each decision to be the unit value. Thus, $\sum_{m} w_{m}^{2}=1, m \in[11,12,13,14,15,16]$ was set for decision No.2, according to Figs. 2 and 3. The first type of initial belief was to set high expected payoffs for the candidate(s) that is (are) consistent with the real interaction matrix of the environment and, at the same time, to set low expected payoffs for other candidates. More specifically, we set the summation of the high expected payoffs to 0.8 and the summation of low expected payoffs to 0.2. Because the real interactions are decisions No.11 and No.14, for decision No.2, the initial expected payoffs for these two candidates were $w_{11}^{2}=w_{14}^{2}=0.8 / 2$, and the initial expected payoffs for other candidates were $w_{12}^{2}=w_{13}^{2}=w_{15}^{2}=w_{16}^{2}=0.2 / 4$. This type of initial belief represents the case in which the agent has a preference about selecting interaction candidates and this preference is consistent with the real environment. Hence, this type will be referred to as "correct initial belief."

The second type of initial belief is opposite to the first one; it indicates the case in which the agent has a wrong perception of the interaction candidates. For instance, the agent may have the perception that decisions No.12 and No.13, rather than No.11 and No.14, are likely to be the interactions of decision No.2. Thus, we assigned high expected payoffs to candidates No.12 and No.13 and low expected payoffs to other candidates. Hence, we set $w_{12}^{2}=w_{13}^{2}=0.8 / 2$ and $w_{11}^{2}=w_{14}^{2}=w_{15}^{2}=w_{16}^{2}=0.2 / 4$. In the simulation, the preferred interactions (in this example, No.12 and No.13) were randomly selected. This wrong perception may result in a wrong perceived interaction matrix that is quite different from the real environment. Hence, this type will be referred to as "incorrect initial belief."

Considering that the agent may not have any perception about the possible interactions, we designed the third type of initial belief, which was to set the same expected payoffs for every interaction candidates, i.e., $w_{m}^{2}=1 / 6, m \in$ $[11,12,13,14,15,16]$. This type will be referred to as "fair initial belief." It should be noted that in a single simulation, only one type will be chosen to be implemented to all decisions.

In this experiment, we designed 18 scenarios with two levels of parameter $X$, three types of initial beliefs, and three types of collaborative search that have been described in Section 3.3. In each scenario, four parameters of the learning behaviors of the agents were selected in the following ranges: reinforcement learning parameters $\phi \in[0.1,0.8], n_{L} \in[1,3]$, and $\tau=0.1$, and the learning frequency parameter $T_{L} \in[5,50]$. Figs. 5 and 6 show representative results pertaining to how the reinforcement learning parameter $\phi$ and $n_{L}$ affect the organizational performance for different initial knowledge. More specifically, these results are based on parameters $X=20 \%$ and $T_{L}=5$, and on the search collaboration Type III.

Figure 5 shows the learning performance of the organization at each learning time period. The learning performance was measured in terms of the correctness of the perceived inter-firm interactions (i.e., the y-axis in each graph). This correctness is defined as the percentage of the correct interactions among the perceived inter-firm interactions (in the light-color area of the interaction matrix). According to the results presented in the three diagrams, the "initial beliefs" are found to be quite influential to the learning performance. The organization appears to have a high learning performance (over 0.9 ) when agents have "correct initial beliefs"; on the other hand, it presents a very low performance (less than 0.05 ) when agents have "incorrect initial beliefs." However, the learning performance in the case of "fair initial beliefs" can be observed to be robust at a medium level (approximately 0.2-0.3). Parameter $\phi$, which indicates the sensitivity to the performance feedback in updating the expected payoffs of the interaction candidates, appears to have the opposite effect on the learning performance in the cases of "correct initial beliefs" and "incorrect initial beliefs." Low $\phi$ values improve the performance when the initial beliefs are correct; however, the performance deteriorates when the initial beliefs are incorrect. High $\phi$ values have an opposite influence. Parameter $n_{L}$, which indicates the number of decisions that are chosen to execute learning at each time, appears to have the same impact in most cases: a high $n_{L}$ can intensify the improvement or the decline of the performance.

Figure 6 shows the search performance of the organization at each search time period. The search performance is measured in terms of the fitness value (according to the real environment landscape) of the proposed strategy of the organization. Comparing the results in the three diagrams, the "initial beliefs" of the agents about the inter-firm interactions can be observed to have similar effects on the search performance and learning performance of the organization. The cases of "correct initial beliefs" could result in high learning and search perfor- 

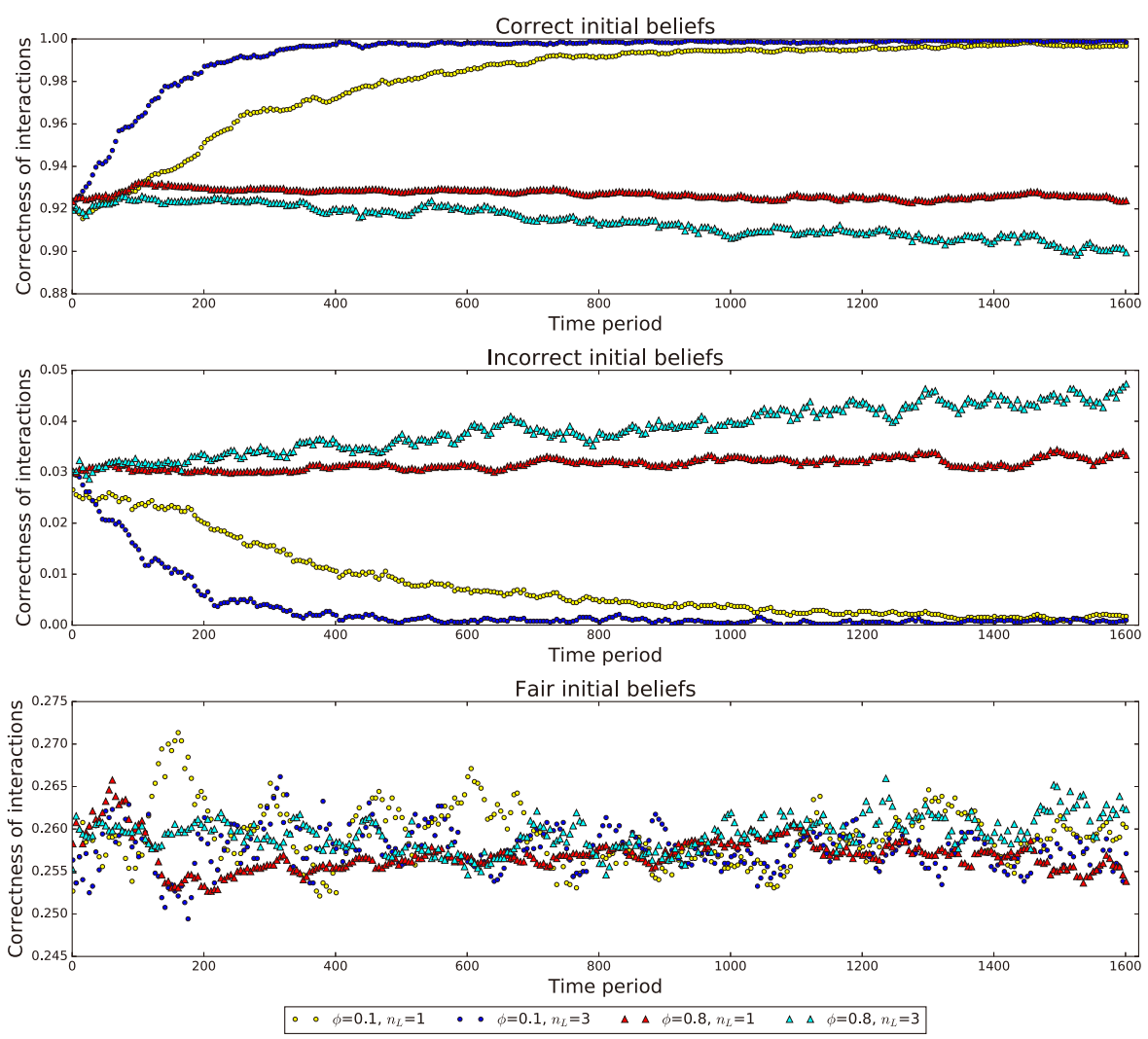

Fig. 5. The learning performance of the organization for different $\phi$ and $n_{L}\left(X=20 \%, T_{L}=5\right.$, search collaboration Type III).
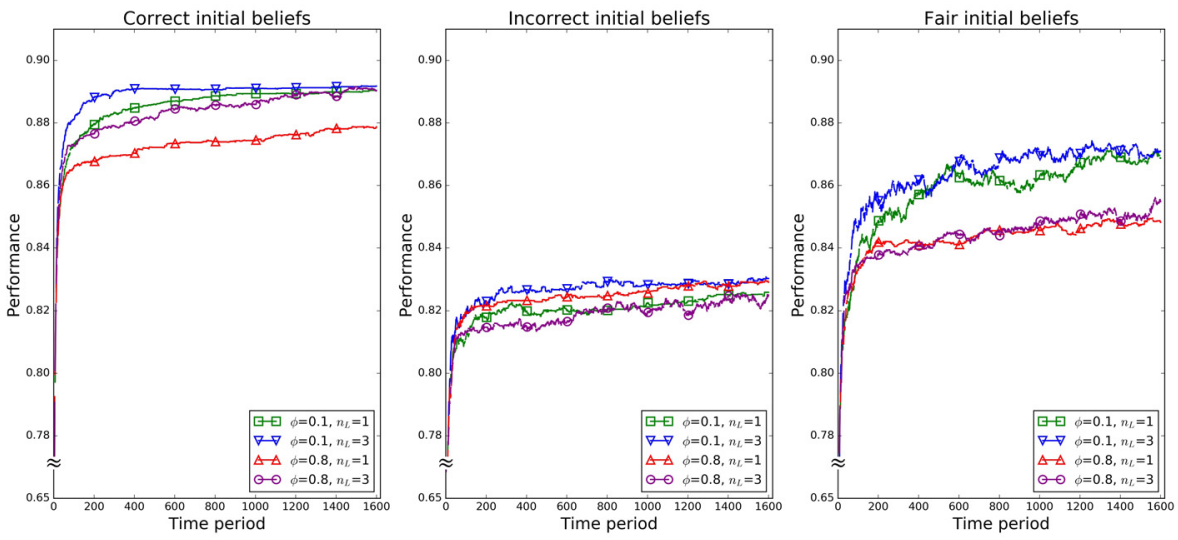

Fig. 6. The search performance of the organization for different $\phi$ and $n_{L}\left(X=20 \%, T_{L}=5\right.$, search collaboration Type III).

mance; however, "incorrect initial beliefs" could lead to low learning and search performance. Lower $\phi$ values may be observed to have a positive effect on the search performance in most cases and higher $n_{L}$ values can intensify this effect. According to the NK model, interactions between the decisions are essential factors in evaluating the fitness of the strategy. More correctly perceived interactions could help agents to evaluate the fitness more properly. Thus, the results of the learning and search performance of the organization are consistent in general.

Figures 7 and $\mathbf{8}$ present the representative results per- taining to the impact of the learning frequency parameter $T_{L}$ to the organizational performance for different initial knowledge. These results are based on parameters $X=20 \%, \phi=0.1$, and $n_{L}=1$, and on the search collaboration Type III. According to Fig. 7, a high $T_{L}$ value, which indicates a large learning time interval or a low learning frequency, could weaken the learning efficiency, unless the agents have "fair initial beliefs" about the inter-firm interactions. Furthermore, a high $T_{L}$ could prevent the organization from presenting a high performance when the agents have "correct initial beliefs" and 

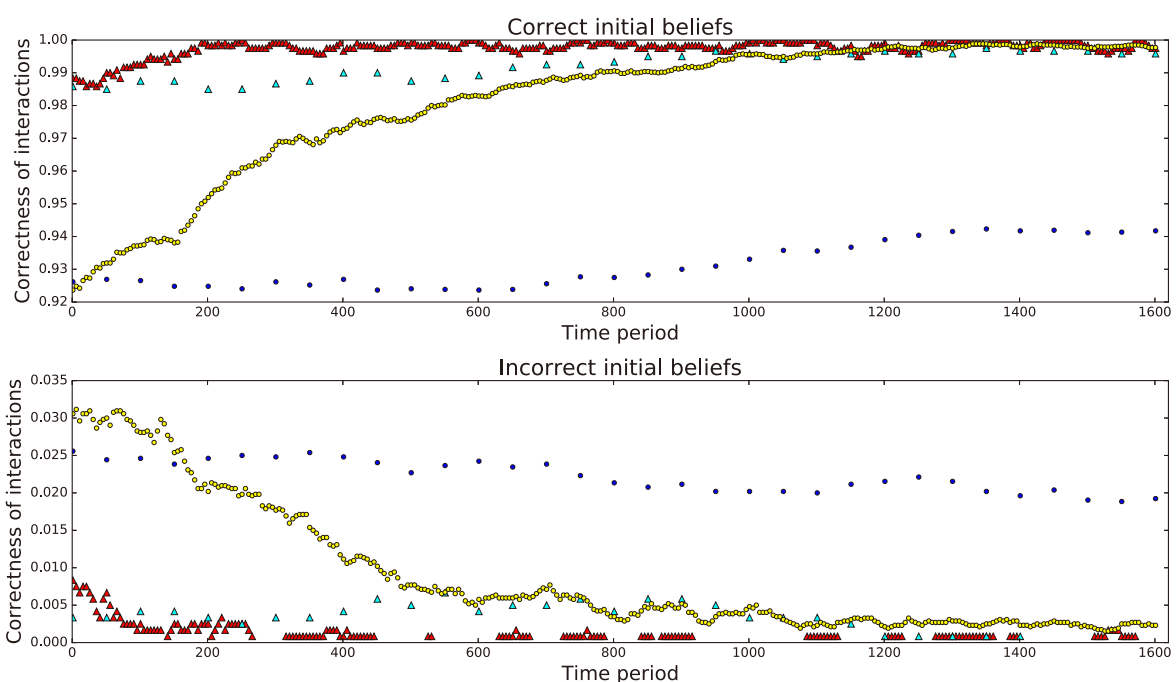

Fair initial beliefs

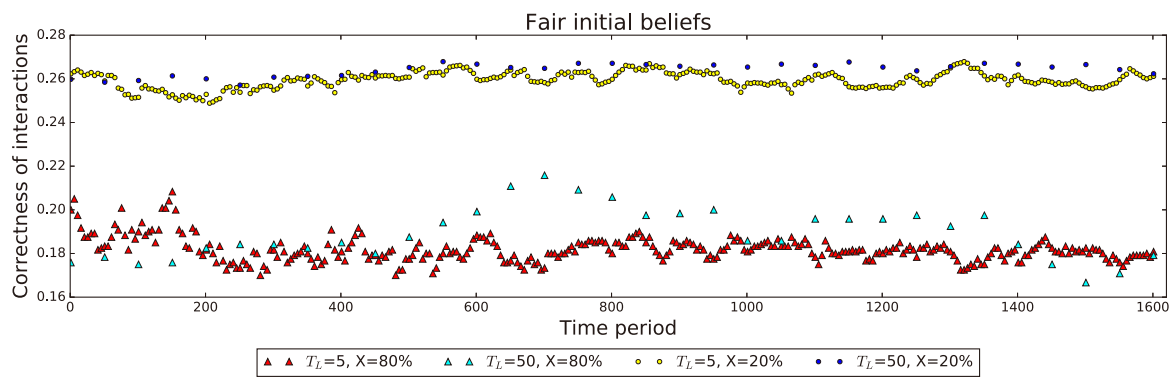

Fig. 7. The learning performance of the organization for different $T_{L}$ and $X\left(\phi=0.1, n_{L}=1\right.$, search collaboration Type III).
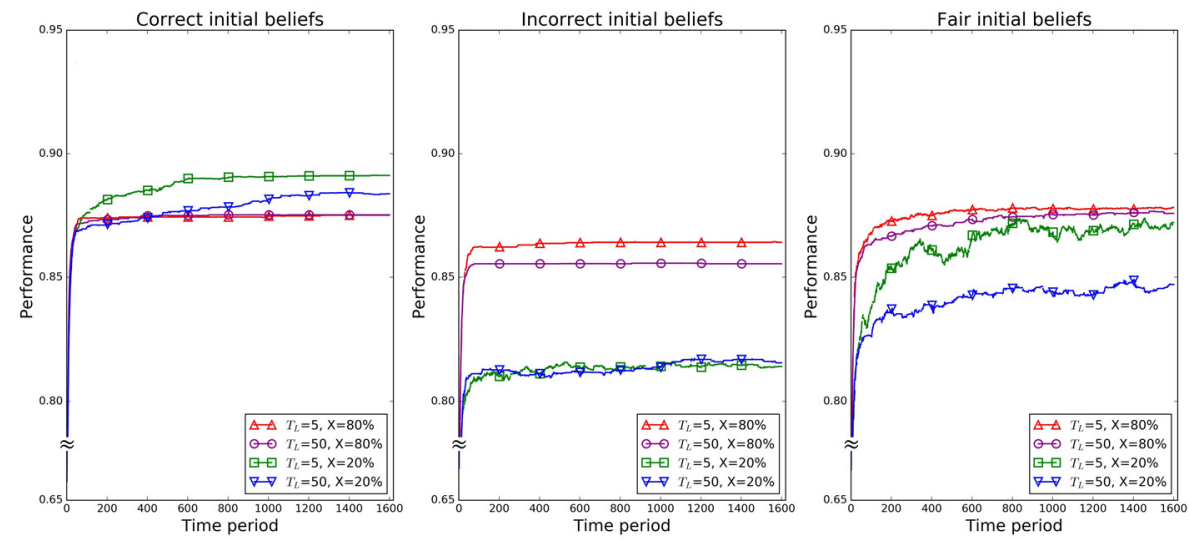

Fig. 8. The search performance of the organization for different $T_{L}$ and $X\left(\phi=0.1, n_{L}=1\right.$, search collaboration Type III).

could protect the organization against low performance when agents have "incorrect initial beliefs." This feature is suitable for both levels of $X$. According to Fig. 8, the search performance of the organization subject to the impact of different "initial beliefs" may be observed to be similar to that in Fig. 6 in the case of $X=20 \%$. Moreover, frequent learning (i.e., low $T_{L}$ ) appears to have a positive influence on the search performance in the cases of "correct initial belief" and "fair initial belief." However, in the case of $X=80 \%$, the search performance of the organization in different scenarios remains at approximately the same level, which may be attributed to the fact that the percentage of the total correct inter-firm interactions is quite high (as a minimum of $80 \%$ ) for three types of "initial beliefs," despite the fact that the percentage of the correct perceived interactions are different. The correctness of interactions is within the range of $[80 \%, 100 \%]$. Thus, the search performance remains at almost the same level.

Consequently, the initial perceptions of the companies regarding the new task environment (i.e., the "initial beliefs" about the inter-firm interactions) are quite influ- 
ential to the performance of the organization during the adaptation process. The organization in which the agents have a correct initial perception of the new environment (i.e., the case of "correct initial belief") is likely to present excellent performance. Furthermore, the adaptation of the organization could be improved by means of a low sensitivity to the performance feedback regarding the update of perceptions (i.e., low $\phi$ ), by making more decisions to conduct learning each time (i.e., large $n_{L}$ ), and via a high learning frequency (i.e., low $T_{L}$ ). Conversely, an organization in which the agents have an incorrect initial perception of the new environment may present a low performance. In this case, a low $\phi$ value with a high $n_{L}$ value could reinforce an incorrect perception of the environment, thus compromising the adaptation process. On the contrary, a high sensitivity to the performance feedback (i.e., high $\phi$ ) with high $n_{L}$ value could effectively revise the perceptions of the agents and help the organization to adapt to the new environment. In general, when agents have no initial perception of the new environment, the organization may present a robust performance with different learning behaviors and it may have a slow yet lasting adaptation process.

\subsection{The Impact of the Search Behaviors on the Per- formance of Adaptation}

The second round of experiments pertained to how the search behaviors of the organization would impact its performance during the adaptation process. In this experiment, we designed several scenarios with two levels of $X$, three types of "initial beliefs" of the agents, and three types of collaborative search behavior. In addition, we released one of the search behaviors, which was referred to as "search radius (parameter $S R$ )" [18]. More specifically, the search radius was defined as the number of decisions that can be changed at each time of search. In Section 3.3, we modeled a base case in which agents could change the value of only one single decision at each time of search $(S R=1)$. In this experiment, we added a more complex case, in which agents could change the values of three decisions simultaneously $(S R=3)$. To focus on the search behaviors, we set that the following learning behavior parameters be fixed: $\phi=0.1, n_{L}=1$, and $T_{L}=5$.

Figures 9 and 10 show the representative results of the effect of different $S R$ and $X$ values. Similar to the previous experiments, we selected the results of search collaboration Type III. According to Fig. 9, the learning performance of the organization is consistent with the result of the previous experiment; in most cases, the search radius has little impact on the learning performance. Nonetheless, the search performance of the organization appears to be quite sensitive to the $S R$, according to Fig. 10. In all cases, a large search radius can significantly enhance the search performance of the organization. Because complex interdependencies exist between decisions, the landscape could be rugged with many local peaks. Agents can easily become trapped in local peaks during the search process. However, an increase in the search radius could reduce the chances of agents becoming trapped in these local peaks [18]. Consequently, an increase in the search radius could efficiently help the organization to adapt to the new environment in order to achieve a higher performance, despite the fact that the company has incomplete knowledge of the new environment.

\section{Conclusions}

In this work, we proposed an agent-based model to study the adaptation of a complex system to an unfamiliar environment by investigating the case of the postacquisition integration of companies. In particular, we modeled the original environment of two companies as two NK landscapes. After the acquisition, the two companies merged, thus inducing environmental change. Considering the bounded rationality, we assumed that the companies have limited knowledge about the new environment. Then, we proposed a coupled learning method over the NK landscape to study the adaptation of the organization. Finally, we designed several experiments based on the proposed model and we discussed the impact of the learning and search behaviors of the organization on the adaptation performance.

The simulation results showed that the initial perceptions of the agents (i.e., the companies) on the new task environment were quite influential on the performance of the entire system during the adaptation process. Correct initial perceptions can help the system to quickly achieve high performance; however, incorrect initial perceptions may prevent the system from achieving high performance. Lack of initial perceptions led to a slow yet robust adaptation process, with a moderate level of performance. Moreover, the behaviors of the agents had different impact on the adaptation performance of the system. The sensitivity to the performance feedback (i.e., parameter $\phi$ ) had opposite effects for different cases of initial perceptions. Agents that had correct initial perceptions about the new environment needed a low sensitivity to the performance feedback when updating their perceptions because low sensitivity could help them to reinforce the correct perceptions. Conversely, agents that had incorrect initial perceptions should opt for a high sensitivity to the performance feedback to revise their incorrect perceptions about the environment. The incentive of the system pertaining to the exploration (i.e., parameter $n_{L}$ ) can adjust the speed of the adaptation of the system. A higher $n_{L}$ value intensified the reinforcement learning effect (including both positive and negative effects) and accelerated the adaptation process of the system. On the other hand, a lower $n_{L}$ value led to a cautious adaptation process. Regarding the learning frequency, a low frequency typically results in a sluggish adaptation process. Moreover, an increase in the search radius was found to be efficient in helping the system to adapt to the new environment and to achieve higher performance, even with the incomplete knowledge about the environment.

In this work, we proposed a coupled learning method 

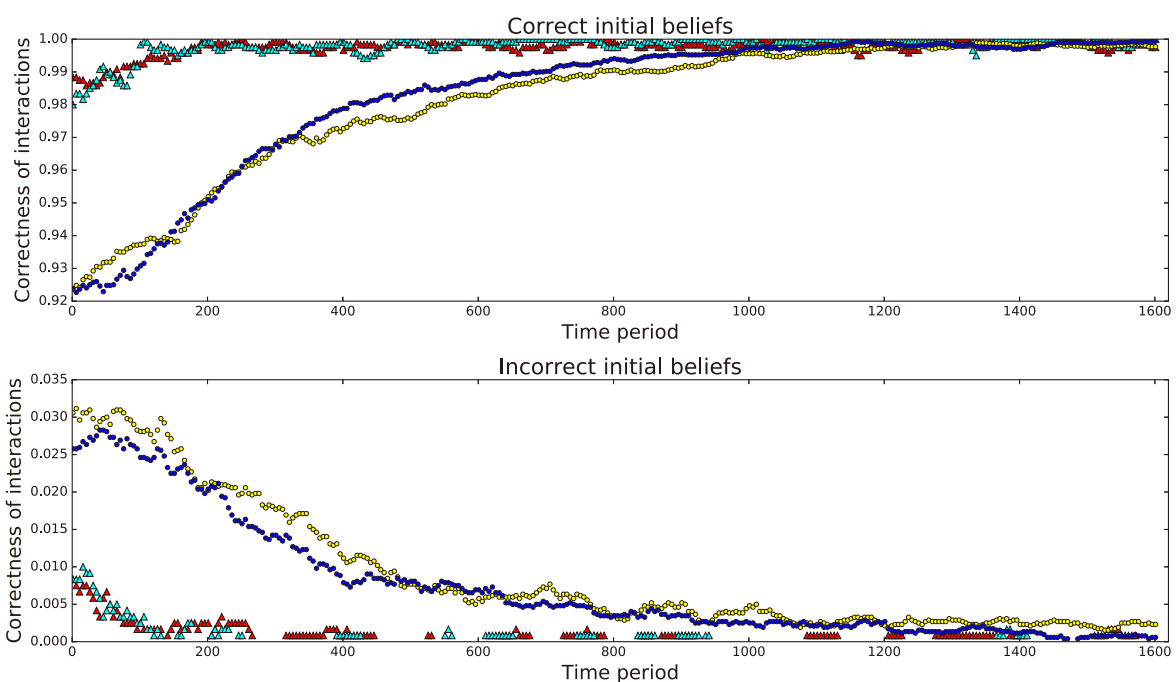

Fair initial beliefs

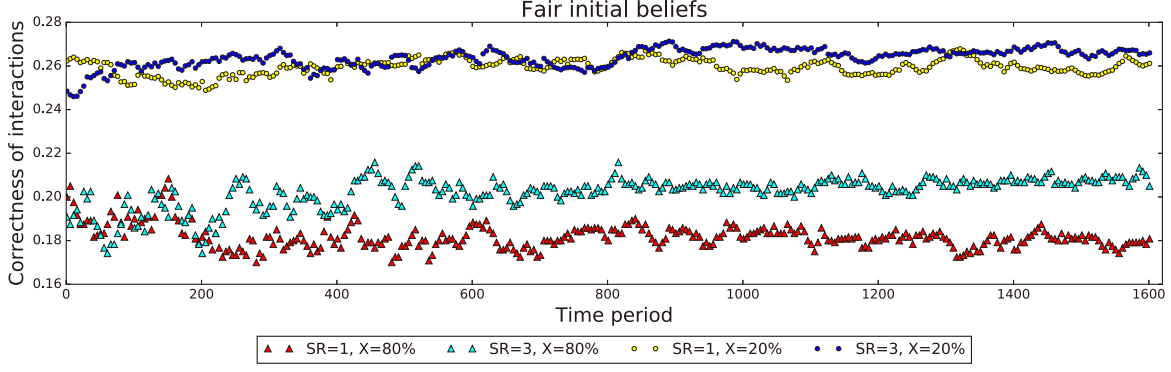

Fig. 9. The learning performance of the organization for different $S R$ and $X\left(\phi=0.1, n_{L}=1, T_{L}=5\right.$, search collaboration Type III).
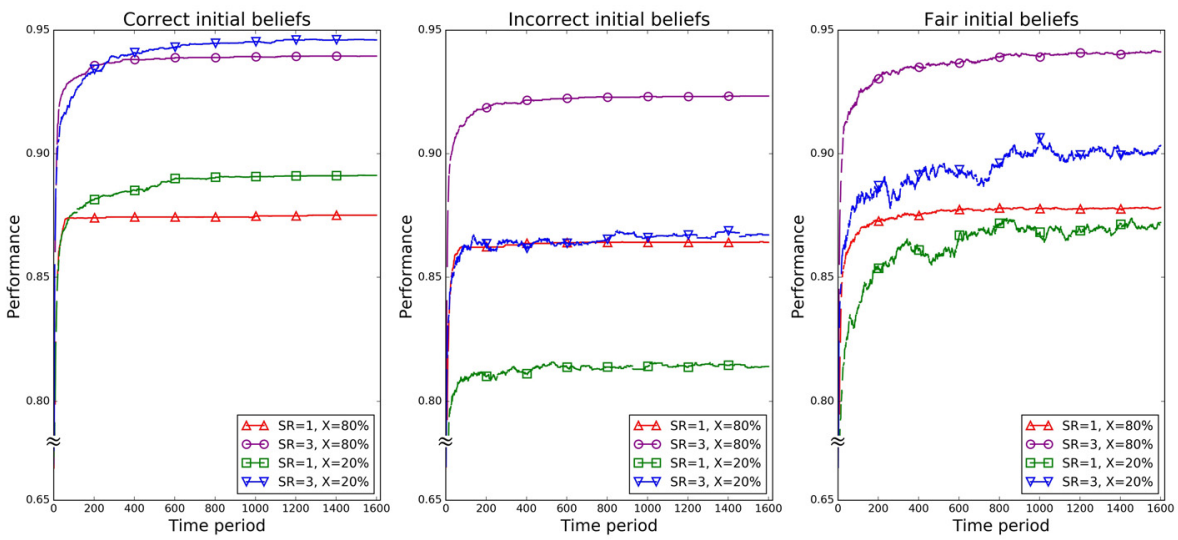

Fig. 10. The search performance of the organization for different $S R$ and $X\left(\phi=0.1, n_{L}=1, T_{L}=5\right.$, search collaboration Type III).

over the NK landscape and it was found to be feasible according to our simulation results. Because we modeled the post-acquisition integration as the reason of environmental change for the companies, the new environment had a relatively special interaction matrix with a fourquadrant pattern, and the limited knowledge of the agents was applied to the inter-firm interactions. However, in our future work, the restrictions set by the interaction pattern can be relaxed with several other research problems or scenarios. As the NK landscape is widely used for the modeling of the complex interactions within or between the system(s), the model we proposed in this work can be modified and applied to several research problems for the purpose of gaining insight to the complex interactions of systems.

\section{References:}

[1] J. H. Holland, "Complex adaptive systems," Daedalus, Vol.121, No.1. pp. 17-30, 1992.

[2] J. H. Holland, "Studying complex adaptive systems," J. of Systems Science and Complexity, Vol.19, No.1, pp. 1-8, 2006.

[3] S. D. Pathak, J. M. Day, A. Nair, W. J. Sawaya, and M. M. Kristal, "Complexity and adaptivity in supply networks: Building supply 
network theory using a complex adaptive systems perspective," Decision Sciences, Vol.38, No.4, pp. 547-580, 2007.

[4] W. P. Millhiser and D. Solow, "How large should a complex system be? An application in organizational teams," Complexity, Vol.12, No.4, pp. 54-70, 2007

[5] R. M. Cyert and J. G. March, "A behavioral theory of the firm," Prentice-Hall, 1963.

[6] S. A. Kauffman and E. D. Weinberger, "The NK model of rugged fitness landscapes and its application to maturation of the immune response," J. of Theoretical Biology, Vol.141, No.2, pp. 211-245, 1989.

[7] J. Uotila, "Punctuated equilibrium or ambidexterity: Dynamics of incremental and radical organizational change over time," Industrial and Corporate Change, Vol.27, No.1, pp. 131-148, 2017.

[8] J. Claussen, T. Kretschmer, and N. Stieglitz, "Vertical scope, turbulence, and the benefits of commitment and flexibility," Management Science, Vol.61, No.4, pp. 915-929, 2015.

[9] S. Yi, T. Knudsen, and M. C. Becker, "Inertia in routines: A hidden source of organizational variation," Organization Science, Vol.27, No.3, pp. 782-800, 2016.

[10] H. A. Simon, "A behavioral model of rational choice," The Quarterly J. of Economics, Vol.69, No.1, pp. 99-118, 1955.

[11] D. A. Levinthal, "Adaptation on rugged landscapes," Management science, Vol.43, No.7, pp. 934-950, 1997.

[12] N. Siggelkow and J. W. Rivkin, "Speed and search: Designing organizations for turbulence and complexity," Organization Science, Vol.16, No.2, pp. 101-122, 2005.

[13] N. Stieglitz, T. Knudsen, and M. C. Becker, "Adaptation and inertia in dynamic environments," Strategic Management J., Vol.37, No.9, pp. 1854-1864, 2016.

[14] S. K. Ethiraj and D. Levinthal, "Bounded rationality and the search for organizational architecture: An evolutionary perspective on the design of organizations and their evolvability," Administrative Science Quarterly, Vol.49, No.3, pp. 404-437, 2004.

[15] D. A. Levinthal and A. Marino, "Three facets of organizational adaptation: Selection, variety, and plasticity," Organization Science, Vol.26, No.3, pp. 743-755, 2015

[16] J. W. Rivkin and N. Siggelkow, "Organizational sticking points on NK landscapes," Complexity, Vol.7, No.5, pp. 31-43, 2002.

[17] J. W. Rivkin and N. Siggelkow, "Patterned interactions in complex systems: Implications for exploration," Management Science, Vol.53, No.7, pp. 1068-1085, 2007.

[18] V. A. Aggarwal, N. Siggelkow, and H. Singh, "Governing collaborative activity: Interdependence and the impact of coordination and exploration," Strategic Management J., Vol.32, No.7, pp. 705-730, 2011.

[19] F. A. Csaszar and J. P. Eggers, "Organizational decision making: An information aggregation view," Management Science, Vol.59, No.10, pp. 2257-2277, 2013

[20] T. Knudsen and D. A. Levinthal, "Two faces of search: Alternative generation and alternative evaluation," Organization Science, Vol.18, No.1, pp. 39-54, 2007.

[21] J. Mihm, C. H. Loch, D. Wilkinson, and B. A. Huberman, "Hierarchical structure and search in complex organizations," Management Science, Vol.56, No.5, pp. 831-848, 2010.

[22] P. C. Haspeslagh and D. B. Jemison, "Managing acquisitions: Creating value through corporate renewal," New York: Free Press, 1991.

[23] J. Birkinshaw, H. Bresman, and L. Hakanson, "Managing the postacquisition integration process: How the human integration and task integration processes interact to foster value creation," J. of Management Studies, Vol.37, No.3, pp. 395-425, 2000.

[24] R. Larsson and S. Finkelstein, "Integrating strategic, organizational, and human resource perspectives on mergers and acquisitions: A case survey of synergy realization," Organization Science, Vol.10, No.1, pp. 1-26, 1999.

[25] A. Zaheer, X. Castaer, and D. Souder, "Synergy sources, target autonomy, and integration in acquisitions," J. of Management, Vol.39, No.3, pp. 604-632, 2013.

[26] B. Uzelac, F. Bauer, K. Matzler, and M. Waschak, "The moderating effects of decision-making preferences on M\&A integration speed and performance," The Int. J. of Human Resource Management, Vol.27, No.20, pp. 2436-2460, 2016.

[27] H. Bresman, J. Birkinshaw, and R. Nobel, "Knowledge transfer in international acquisitions," J. of Int. Business Studies, Vol.30, No.3, pp. 439-462, 1999.

[28] J. Gammelgaard, K. Husted, and S. Michailova, "Knowledge sharing behavior and post-acquisition integration failure," CKGWP 6 , pp. 24-41, 2004

[29] A. L. Ranft, "Knowledge preservation and transfer during postacquisition integration," Advances in Mergers and Acquisitions, Emerald Group Publishing Limited, pp. 51-67, 2006.
[30] E. Gomes, D. N. Angwin, Y. Weber, and S. Yedidia Tarba, "Critical success factors through the mergers and acquisitions process: Revealing pre-and post-M\&A connections for improved performance," Thunderbird Int. Business Review, Vol.55, No.1, pp. 13-35, 2013.

[31] G. Gavetti, D. A. Levinthal, and J. W. Rivkin, "Strategy making in novel and complex worlds: The power of analogy," Strategic Management J., Vol.26, No.8, pp. 691-712, 2005.

[32] P. Puranam and M. Swamy, "How initial representations shape coupled learning processes," Organization Science, Vol.27, No.2, pp. 323-335, 2016.

[33] M. Jafari Songhori, M. S. Jalali, and T. Terano, "The effects of teams' initial characterizations of interactions on product development performance," Simulation Conf. (WSC), 2017 Winter, pp. 1383-1394, 2017.

[34] R. S. Sutton and A. G. Barto, "Reinforcement learning," MIT Press Cambridge, 1998.

[35] J. Denrell and J. G. March, "Adaptation as information restriction: The hot stove effect," Organization Science, Vol.12, No.5, pp. 523$538,2001$.

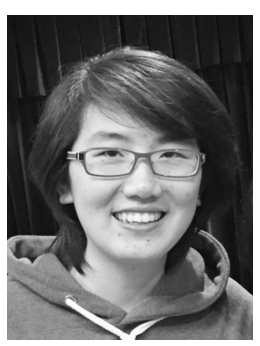

Name:

Jing Su

\section{Affiliation:}

Doctoral Student, Tokyo Institute of Technology

Address:

4259 Nagatsuta-cho, Midori-ku, Yokohama, Kanagawa 226-8502, Japan

Brief Biographical History:

2010 Received B.E. degree from Beihang University

2013 Received M.E. degree from Beihang University

Main Works:

- Her research interests include agent-based modeling and simulation, computational organization theory, evolutionary computation, and air traffic management. 


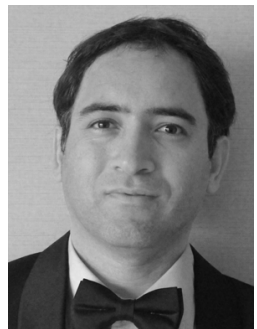

Name:

Mohsen Jafari Songhori

\section{Affiliation:}

Research Fellow, Department of Health Technology and Services Research, Technical Medical Center, University of Twente

\section{Address:}

Ravelijn 5151, P.O. Box 217, 7500 AE Enschede, The Netherlands

Brief Biographical History:

2014 Ph.D. in Technology and Operations Management

2014-2016 JSPS Fellow at Tokyo Institute of Technology

2017- Postdoctoral Fellow at University of Twente

Main Works:

- His research interests include, but not limited to computational and analytical research in product development, technology management, operations management, and innovation.

- M. Jafari Songhori and T. Terano, "How to Form Product Development Teams? Effects of Organizational and Product Related Factors," SSRN.

- M. Jafari Songhori and J. Nasiry, "Organizational Structure, Subsystem Centrality, and Misalignments in Complex NPD Projects," SSRN.

Membership in Academic Societies:

- The Institute for Operations Research and the Management Sciences (INFORMS), Member

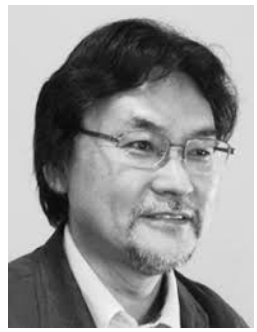

\section{Name:}

Takao Terano

\section{Affiliation:}

Researcher, AIST-Tokyo Tech Real World BigData Computation Open Innovation Laboratory (RWBC-OIL), National Institute of Advanced Industrial Science and Technology

\section{Address:}

2-12-1 Ookayama, Meguro-ku, Tokyo 152-8550, Japan

Brief Biographical History:

1976 Received B.A. degree from the University of Tokyo

1978 Received M.A. degree from the University of Tokyo

1978-1989 Scientist at the Central Research Institute of the Electric Power Industry

1991 Received Doctor of Engineering Degree from Tokyo Institute of

Technology

1990-2004 Professor at University of Tsukuba

2004-2018 Professor at Tokyo Institute of Technology

Main Works:

- His research interests include, but not limited to genetic algorithm-based machine learning, case-based reasoning, analogical reasoning, distributed artificial intelligence, cooperative agents, computational organization theory, and knowledge system development methodology.

\section{Membership in Academic Societies:}

- Member, the editorial board of major Artificial Intelligence-related academic societies in Japan

- The Institute of Electrical and Electronics Engineers (IEEE), Member

- Association for the Advancement of Artificial Intelligence (AAAI),

Member

- Association for Computing Machinery (ACM), Member

- Pacific-Asian Association for Agent-based Approach in Social Systems

Sciences (PAAA), President 